\title{
Dopamine or opioid stimulation of nucleus accumbens similarly amplify cue-triggered 'wanting' for reward: entire core and medial shell mapped as substrates for PIT enhancement
}

\author{
Susana Peciña ${ }^{1}$ and Kent C. Berridge ${ }^{2}$ \\ ${ }^{1}$ Department of Behavioral Sciences, University of Michigan-Dearborn, Dearborn, MI, USA \\ ²Department of Psychology, University of Michigan, Ann Arbor, MI, USA
}

Keywords: amphetamine, DAMGO, fos mapping, liking, motivation, rat

\begin{abstract}
Pavlovian cues [conditioned stimulus (CS+)] often trigger intense motivation to pursue and consume related reward [unconditioned stimulus (UCS)]. But cues do not always trigger the same intensity of motivation. Encountering a reward cue can be more tempting on some occasions than on others. What makes the same cue trigger more intense motivation to pursue reward on a particular encounter? The answer may be the level of incentive salience ('wanting') that is dynamically generated by mesocorticolimbic brain systems, influenced especially by dopamine and opioid neurotransmission in the nucleus accumbens (NAc) at that moment. We tested the ability of dopamine stimulation (by amphetamine microinjection) vs. mu opioid stimulation [by d-Ala, nMePhe, Glyol-enkephalin (DAMGO) microinjection] of either the core or shell of the NAc to amplify cue-triggered levels of motivation to pursue sucrose reward, measured with a Pavlovian-Instrumental Transfer (PIT) procedure, a relatively pure assay of incentive salience. Cue-triggered 'wanting' in PIT was enhanced by amphetamine or DAMGO microinjections equally, and also equally at nearly all sites throughout the entire core and medial shell (except for a small far-rostral strip of shell). NAc dopamine/opioid stimulations specifically enhanced CS+ ability to trigger phasic peaks of 'wanting' to obtain UCS, without altering baseline efforts when CS+ was absent. We conclude that dopamine/opioid stimulation throughout nearly the entire NAc can causally amplify the reactivity of mesocorticolimbic circuits, and so magnify incentive salience or phasic UCS 'wanting' peaks triggered by a CS+. Mesolimbic amplification of incentive salience may explain why a particular cue encounter can become irresistibly tempting, even when previous encounters were successfully resisted before.
\end{abstract}

\section{Introduction}

In drug addiction, binge eating and other compulsive reward-seeking disorders, encounters with learned cues (Pavlovian conditioned stimulus or $\mathrm{CS}+$ ) can trigger intense urges to obtain an associated reward (unconditioned stimulus or UCS). However, the motivational power of cues fluctuates across encounters. A cue may be successfully resisted many times, yet on another occasion trigger irresistible temptation and relapse. What underlies a sudden surge in Pavlovian motivational power?

Fluctuations in the level of motivation elicited by reward cues (i.e. changes cue-triggered 'wanting') may be mediated by changes in reactivity of mesocorticolimbic brain circuits involving the nucleus accumbens (NAc), which dynamically compute incentive salience for a cue (Everitt \& Robbins, 2005; Smith et al., 2011; Wassum et al., 2011; Berridge, 2012; Saunders \& Robinson, 2012). The level of 'wanting' depends on the brain's mesocorticolimbic

Correspondence: Dr S. Peciña, as above.

E-mail: pesu@umich.edu

Received 30 July 2012, revised 27 January 2013, accepted 30 January 2013 reactivity state at the moment, as well as on the stable learned association value of the $\mathrm{CS}+$.

In animal studies, cue-triggered 'wanting' for reward can be modeled using Pavlovian-Instrumental Transfer (PIT) procedures (Walker, 1942; Estes, 1943; Dickinson et al., 2000; Corbit et al., 2007; Bray et al., 2008; Crombag et al., 2008; Talmi et al., 2008; O'Connor et al., 2010), which is a relatively pure measure of incentive salience. PIT can help answer if neurochemical stimulations of mesocorticolimbic circuits directly enhance the ability of a $\mathrm{CS}+$ to trigger stronger peaks of motivation to obtain its UCS reward. The experimental design and patterns of behavioral elevation in PIT help isolate incentive salience by excluding alternative explanations based on enhancements of other psychological processes (e.g. of primary UCS hedonic impact, Pavlovian S-R habits between $\mathrm{CS}+$ and responses, etc.). Previous studies showed that dopamine and some other manipulations of the NAc can alter the magnitude of PIT in ways that reflect changes in cue-triggered 'wanting' (Dickinson et al., 2000; Wyvell \& Berridge, 2000, 2001; Holland \& Gallagher, 2003; Corbit \& Balleine, 2005; Peciña et al., 2006; Lex \& Hauber, 2008; Mahler \& Berridge, 2009; Pielock 
et al., 2011; Wassum et al., 2011; Laurent et al., 2012; Ostlund \& Maidment, 2012).

Beyond dopamine, mu opioid stimulation in the NAc may similarly enhance incentive motivation for reward, at least as measured in other behavioral tests, such as food intake, instrumental performance, etc. (Mucha \& Iversen, 1986; Bakshi \& Kelley, 1993; Van Ree et al., 2000; Zhang et al., 2003; Bodnar, 2004; Levine \& Billington, 2004; Peciña \& Berridge, 2005; Baldo \& Kelley, 2007; Barbano et al., 2009; Taha et al., 2009; Smith et al., 2011). Does this imply that opioid stimulation of the NAc would enhance PIT as well as dopamine stimulation? Here we mapped NAc substrates for magnifying incentive salience as measured by PIT. We compared dopamine and mu opioid forms of neurochemical stimulation in enhancing cue-triggered 'wanting' for reward. The results show that nearly the entire medial shell and the entire core can similarly mediate dopamine and opioid enhancements of incentive salience, and that dopamine and opioid enhancements are comparable in their magnitude at most NAc sites.

\section{Materials and methods}

\section{Overview}

We aimed to compare the effects of mu opioid [via d-Ala, nMe-Phe, Glyol-enkephalin (DAMGO) microinjections] vs. dopamine (via amphetamine microinjections) stimulations in the NAc at localized microinjection sites approximately in either NAc core or NAc medial shell. These anatomical sites and neurochemical stimulations were compared for ability to specifically amplify CS+-triggered pulses of incentive salience attributed to a central UCS representation, as measured through a PIT test designed to isolate temporal peaks of cue-triggered 'wanting' to obtain a sweet reward.

In a PIT test, rats press instrumentally but in extinction on a lever that they previously learned would earn sucrose pellets. A 30-s auditory Pavlovian $\mathrm{CS}+$, which rats previously learned in separate Pavlovian CS-UCS training sessions would predict sucrose as the UCS, is presented intermittently. The short $\mathrm{CS}+$ is presented every few minutes, in alternation with a different control CS - sound that predicts nothing. It is important to note that rats have never experienced the CS+ while they were lever pressing before the PIT test (because Pavlovian and instrumental training sessions were always separate). At all other periods, no CS is present (baseline instrumental performance). To be interpreted as incentive salience enhancement, magnifications of motivated behavior by NAc stimulation must be expressed as phasic pulses that come and go with the presence of the CS+ cue, even though the drug remains in the NAc more constantly during the test session. This PIT design precludes a potentiated S-R habit explanation of drug-induced enhancements of lever pressing during a $\mathrm{CS}+$, because the response of lever pressing has never been a habit associated with the $\mathrm{CS}+$ as a stimulus (that is, the lever was never pressed in the presence of a CS+ during training). The phasic temporal pattern of PIT enhancements precludes explanations as stable drug-induced elevations of stress, motor arousal or cognitive expectations of future reward, because all of those would be relatively constant throughout the session, whereas the enhancements last only $1 \mathrm{~min}$ and are bound to the 30-s presence of each CS+. Hedonic reward enhancement by opioid/ dopamine microinjections is additionally precluded as an explanation for enhanced pursuit of sucrose (i.e. 'wanting' more because of 'liking' more) because the PIT test is held in extinction, without any delivery of sucrose reward or UCS, so that sucrose is never received during the test while a drug is in the brain.

\section{Subjects}

Experimentally naive Sprague-Dawley rats $(n=55$ males born at the University of Michigan; weight $=250-350 \mathrm{~g}$ at the beginning of the experiment; maintained on 15-20 g of daily chow and ad libitum water throughout the experiment) were housed in pairs in plastic tub cages under a reverse 12-h light cycle (lights off at 07:00 h). All of the following experimental procedures were approved by the University Committee on the Use and Care of Animals at the University of Michigan, and were carried out according to the Guidelines laid down by the NIH in the USA.

Training and testing were conducted during the dark phase when rats were active. Rats received surgery for implantation of microinjection cannulae in the NAc, and one group $(n=45)$ was used for behavioral testing. To help map localization of function, we additionally obtained Fos plume measurements of the maximum local diameter spread of amphetamine or DAMGO impact on neurons surrounding the microinjection site. Fos plumes were measured in a separate group after a single drug microinjection under similar test conditions $(n=10)$. A separate group was used because Fos plumes shrink over successive microinjection repetitions, meaning that the maximal diameter of drug impact can most accurately be measured after a rat's first and only drug microinjection (Richard \& Berridge, 2011). Fos plume diameter data were integrated with behavioral effect data produced at microinjection sites to create function maps similarly to our previous studies (Peciña \& Berridge, 2000, 2005; Smith \& Berridge, 2005; Reynolds \& Berridge, 2008; Richard \& Berridge, 2011).

\section{Test chambers}

Training and testing took place in computerized operant chambers (Med Associates, St Albans, VT, USA). Each chamber contained a sucrose cup (with photobeam entry detector), two automated levers that could be inserted through the wall into the chamber and audio speaker modules (clicker and tone). A 3-W, 24-V house-light mounted on the top-center of the wall opposite the magazine provided illumination. Sound-attenuating boxes equipped with ventilation fans masked external noise. A computer equipped with MEDPC software (Med Associates) recorded the number of active and inactive lever presses and food cup entries.

\section{PIT training procedures: instrumental training (days $1-19)$}

Rats were initially trained on a variable interval (VI) schedule (with ascending levels of responding demand) to press one lever for sucrose pellet reward. Daily instrumental training sessions (30 min) began with the illumination of a red house-light and extension of a lever, and ended with the retraction of the levers and dimming of the house-light. Two days before tests, rats were twice given 20 sucrose pellets overnight in their home cages to familiarize them with the reward and overcome neophobia (days 1 and 2). To begin training, all rats initially received two sessions of magazine training (days 3 and 4) in which 20 deliveries of a single 45-mg sucrose pellet (Formula F; P.J. Noyes, Lancaster, PA, USA) were dispensed on a fixed time (FT) 1-min schedule of reinforcement. Rats were then shifted to a VI-1 instrumental reinforcement schedule (each reward was delivered on average every $1 \mathrm{~s}$ after at least one lever press on the active lever; days 5-7). A discriminative contingency ensured that presses on one lever in the operant chamber produced sucrose pellets (active lever), whereas presses on the other lever did not 
(control lever, which served as a control measure for any indiscriminate increase in pressing due to general sensorimotor activation). Active and inactive lever assignments were balanced across rats. The schedule of reinforcement for the active lever was progressively increased to VI-5 (days 8-10), VI-10 (days 11-13), VI-30 (days 1416) and VI-45 (days 17-19; Fig. 1).

\section{Pavlovian training (days 20-34)}

Pavlovian conditioning took place after instrumental training was finished. Both levers were absent from the chambers during Pavlovian conditioning to prevent adventitious instrumental reinforcement of lever pressing during the cue, and to prevent any stimulusresponse habit formation between cue and instrumental response (i.e. avoiding pairing of $\mathrm{CS}+$ with a response of lever pressing). Either an auditory pulsed tone $(2.9 \mathrm{kHz}, 60 \mathrm{~dB}, 0.5 \mathrm{~s}$ on/off $)$ or a clicker sound $(60 \mathrm{~dB})$, each presented in a block lasting $30 \mathrm{~s}$, was arbitrarily designated as $\mathrm{CS}+$; the other sound was designated as $\mathrm{CS}-$ for that individual rat. Assignment of $\mathrm{CS}+$ and $\mathrm{CS}-$ was balanced across rats.

During 14 daily Pavlovian training sessions, rats received 10 pairings of the CS+ (30-s duration) followed by immediate delivery of three sucrose pellets (UCS). Similar to previous PIT studies, the CS - was to serve as a control stimulus that was essentially irrelevant to reward rather than to become either a CS+ or a strong conditioned inhibitor of responding (Holland, 2004). Therefore, the CS - (30-s duration) was presented twice during the last three Pavlovian sessions (at the middle and end of each session) and was never followed by sucrose pellets. Nose entries into the sucrose dish were monitored by photobeam detector, and all rats developed a discriminative Pavlovian approach conditioned response (CR) toward the sucrose dish, elicited by the CS + during training but not by the CS- (CR difference scores were calculated to verify acquisition as approaches during 30-s CS minus approaches during 30-s baseline immediately before that CS). All behavior was videorecorded by a camera beneath the transparent floor of the chamber for subsequent slow motion analysis.

\section{Microinjection cannula surgery}

Surgery was carried out after training was finished. Rats were pretreated with $0.1 \mathrm{~mL}$ of atropine sulfate, anesthetized with a mixture of ketamine $\mathrm{HCl}(80 \mathrm{mg} / \mathrm{kg}$, i.p.) and xylazine $(5 \mathrm{mg} / \mathrm{kg})$, and stereotaxically implanted with bilateral 23 -gage guide cannulae targeted at different rostrocaudal and dorsoventral levels of the NAc. A slanted skull position was used, with the incisor bar set at $+5 \mathrm{~mm}$ above interaural zero to achieve a slanted cannula angle and avoid penetrating the lateral ventricles.

Cannulae coordinates were staggered across rats so that as a whole the group's placements filled both the medial shell $(n=23)$ and the core $(n=15)$ of the NAc. For each individual rat, the left and right coordinates bilaterally targeted the same site in either the core or shell, and were symmetrical for that point on both sides of the brain. NAc shell coordinates ranged from $\mathrm{AP}+3.3$ to $+1.8 \mathrm{~mm}$ anterior to bregma, all $\pm 0.9 \mathrm{~mm}$ lateral to the midline, and from
-5.5 to $-7.0 \mathrm{~mm}$ ventral to the skull surface $(2.0 \mathrm{~mm}$ above the injection site). NAc core coordinates ranged from $\mathrm{AP}+3.3$ to $+1.8 \mathrm{~mm}$ anterior to bregma, $\pm 1.9 \mathrm{~mm}$ lateral to the midline, and from -5.5 to $-6.5 \mathrm{~mm}$ ventral to the skull surface $(2.0 \mathrm{~mm}$ above the injection site; Paxinos \& Watson, 1996). Microinjection guide cannulae were anchored with skull screws and cranial cement, and wire stylets were used to prevent cannula occlusion. Rats were allowed to recover for 7 days before testing. To serve as anatomical controls for diffusion, seven additional rats were implanted in sites outside the NAc core or medial shell, either in the neostriatum dorsal to the NAc $(n=2)$, lateral shell (lateral to the most ventral part of the medial shell and ventral to the core; $n=2$ ) or lateral olfactory tubercle (ventral to the medial shell; $n=3$ ).

\section{Pre-test instrumental retraining and extinction pre- exposure (days 42-45)}

After 1-week recovery from surgery, rats were given two additional instrumental training sessions (days 42 and 43) prior to testing to reestablish instrumental lever pressing (VI 45-s schedule), followed by two instrumental extinction days (days 44 and 45) with levers present but no sucrose to habituate animals to the extinction condition (Corbit \& Balleine, 2005).

\section{Drugs and microinjections (days 46-50)}

On test days, rats received bilateral microinjections of vehicle (sterile isotonic saline, $0.2 \mu \mathrm{L}$ per side), DAMGO $(0.05 \mu \mathrm{g} / 0.2 \mu \mathrm{L}$ per side; Sigma, St Louis, MO, USA) or amphetamine sulfate $(20 \mu \mathrm{g} /$ $0.2 \mu \mathrm{L}$ per side; Sigma). Every rat received all three microinjection conditions, on separate days in a counterbalanced order. Doses were chosen based on previous studies (Wyvell \& Berridge, 2000, 2001; Peciña \& Berridge, 2005; Peciña et al., 2006). Microinjections were made with a stainless-steel injector cannula (29-gage), extending $2.0 \mathrm{~mm}$ beyond the ventral tip of the guide, and attached to a syringe pump via PE-20 tubing. Rats were gently hand-held during the bilateral microinjections of drugs or vehicle into the NAc $(0.2 \mu \mathrm{L}$ at a rate of $0.2 \mu \mathrm{L} / \mathrm{min}$ via syringe pump). After infusion, the injectors remained in place for an additional $60 \mathrm{~s}$ to allow for drug diffusion before the obturators were replaced. We note in passing that our amphetamine microinjections in the rostral shell were most likely to have produced selective elevation in synaptic dopamine (and not norepinephrine), whereas caudal sites may have elevated both, because dopamine terminals reach throughout the NAc to sites in the rostral and caudal shell and core (whereas norepinephrine terminals are more restricted to the caudal medial shell; Berridge et al., 1997; Ikemoto, 2007). Rats always remained in their home cages during the 48-h intervals between tests.

\section{PIT test of cue-triggered incentive motivation (days 46-50)}

Phasic peaks in lever pressing that were triggered by sudden presentations of the associated $\mathrm{CS}+$ (cue-triggered incentive motivation) were measured in the pure conditioned incentive paradigm based on

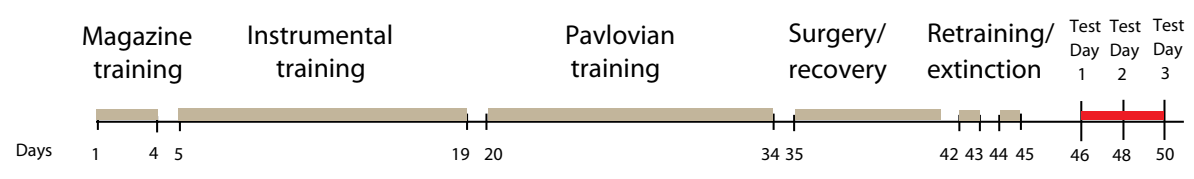

FIG. 1. Experimental timeline for the PIT paradigm. The PIT paradigm included habituation, Pavlovian and instrumental learning, extinction and testing. 
general PIT designed to isolate incentive salience that is triggered by a CS+ and attributed to a central representation of its UCS reward (Wyvell \& Berridge, 2000, 2001; Peciña et al., 2006). On PIT test days, lever pressing was tested with both levers present: the active lever that was previously associated with sucrose reward; and the non-active lever (as control measure for general motor activation). Testing was conducted under extinction conditions in the absence of any sucrose reward. In each test session, the CS + and $\mathrm{CS}-$ were each presented four times for $30 \mathrm{~s}$ each presentation (first $\mathrm{CS}+$ or $\mathrm{CS}-$ presented at $2.5 \mathrm{~min}$; FT 4-min interval; alternating $\mathrm{CS}+/ \mathrm{CS}-$ order).

On each of three test days rats were given bilateral microinjections of vehicle $(0.2 \mu \mathrm{L})$, DAMGO $(0.5 \mu \mathrm{g} / 0.2 \mu \mathrm{L})$ or amphetamine $(20 \mu \mathrm{g} / 0.2 \mu \mathrm{L})$. The order of microinjection was counterbalanced across rats, and test days were spaced $48 \mathrm{~h}$ apart. Immediately after drug microinjections, rats were placed in the instrumental chambers. Instrumental performance was assessed under extinction conditions, so no sucrose pellets were given at any time during the test. Presses on both previously-active and previously-inactive levers were recorded automatically during each $30-\mathrm{s} \mathrm{CS}+$ and $\mathrm{CS}-$ presentation, during the 30 -s period immediately preceding each $\mathrm{CS}+/ \mathrm{CS}-$ (baseline) and during the 30 -s period immediately after each cue presentation. Sucrose dish entries were also recorded during these periods. All behavior was videotaped for subsequent confirmation and analysis.

\section{Fos plume identification and mapping procedures}

A split-and-recombine design similar to previous studies was used to integrate Fos plume data on diameter with behavioral data on the causal effects of drug microinjections on PIT responding (Peciña \& Berridge, 2000, 2005; Smith \& Berridge, 2005). This procedure avoids overly precise localization of function mistakes that would result from underestimation of drug spread based on plumes shrunk by consequences of previous microinjections (e.g. gliosis), because impact is measured in Fos plumes induced by the very first drug microinjection when impact is maximal (Richard \& Berridge, 2011). Ninety minutes after DAMGO, amphetamine or vehicle microinjection, rats were deeply anesthetized with sodium pentobarbital prior to transcardial perfusion. Brains were removed, fixed, sliced and processed for Fos immunofluorescence or Cresyl violet using procedures previously described. DAMGO-induced Fos plumes were identified by comparison with equivalent control sites after vehicle or no microinjections. Maps were created based on the radius of Fos elevation zones, which determined the size of each map symbol. The color of each symbol was determined by the behavioral consequences of drug microinjections at that site measured in PIT.

\section{Statistical analysis of PIT effects}

Repeated-measures ANOVAs were used to determine amphetamine and DAMGO effects on PIT. ANOvas were used to examine the effects of amphetamine (20 mg) and DAMGO $(0.05 \mu \mathrm{g})$ on increases from baseline levels of lever pressing for sucrose during $\mathrm{CS}+$ and $\mathrm{CS}-$ presentations. Additional repeated-measures ANOvAS were employed to examine the specificity of PIT enhancements to cue periods (cue vs. baseline): to the $\mathrm{CS}+$ cue in particular $(\mathrm{CS}+\mathrm{vs}$. $\mathrm{CS}-$ ) and to the sucrose lever (sucrose vs. control lever). The effects of amphetamine and DAMGO in the NAc core, shell and control structures were analysed separately. When significant differences were found, data were further analysed with one-way ANOVA or by Kruskal-Wallis one-way ANOVA on the ranks if data were not normal, followed by Bonferroni post hoc tests.

\section{Results}

\section{Fos plume identification of local impact spread}

DAMGO microinjections $(0.05 \mu \mathrm{g} / 0.2 \mu \mathrm{L})$ produced elevated Fos plumes of approximately $0.5 \mathrm{~mm}^{3}$ volume around the microinjection tip. The plumes contained small centers of $0.03 \mathrm{~mm}^{3}$ volume ( $0.2 \pm 0.03 \mathrm{~mm}$ radius), where Fos expression was intensely elevated $>5$ times over control levels (compared with vehicle microinjection levels), surrounded by a larger halo of $0.5 \mathrm{~mm}^{3}$ volume where Fos was merely doubled over vehicle control levels (0.5 mm radius; Fig. 2). Amphetamine microinjections $(20 \mu \mathrm{g} /$ $0.2 \mu \mathrm{L}$ ) produced similar approximately $0.6 \mathrm{~mm}^{3}$ Fos plumes, containing slightly smaller centers of $0.004 \mathrm{~mm}^{3}$ volume $(0.1 \pm 0.05 \mathrm{~mm}$ radius) for $>4$ times Fos elevations, surrounded by a comparable halo of approximately $0.6 \mathrm{~mm}^{3}(0.6 \pm 0.04 \mathrm{~mm}$ radius; Fig. 2) of doubled Fos expression. These measurements for Fos plume diameters were used to set the size of symbols for microinjection site effects on PIT enhancement shown in all function maps. All other data (e.g. symbol color reflecting behavioral PIT increase) shown in function maps were obtained from the behavioral PIT tests, and reflect enhancements produced by amphetamine/DAMGO microinjection (relative to control vehicle levels measured in the same rat).

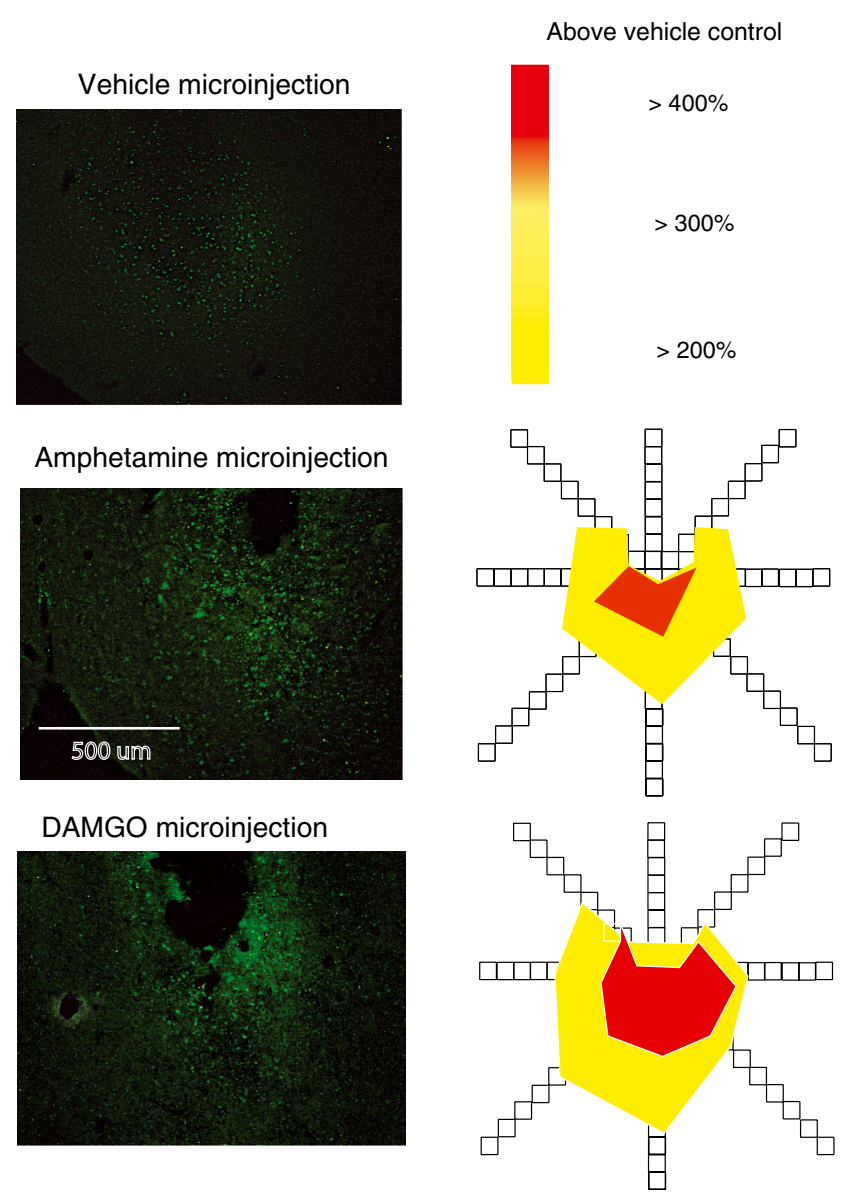

FIG. 2. Fos plume diameters show the spread of drug impact on local NAc neurons. Plumes of elevated Fos expression surrounded microinjections of amphetamine or DAMGO. The average diameter of Fos plumes measured after a single microinjection of each drug was used to set the diameters of site symbols used in function maps. 


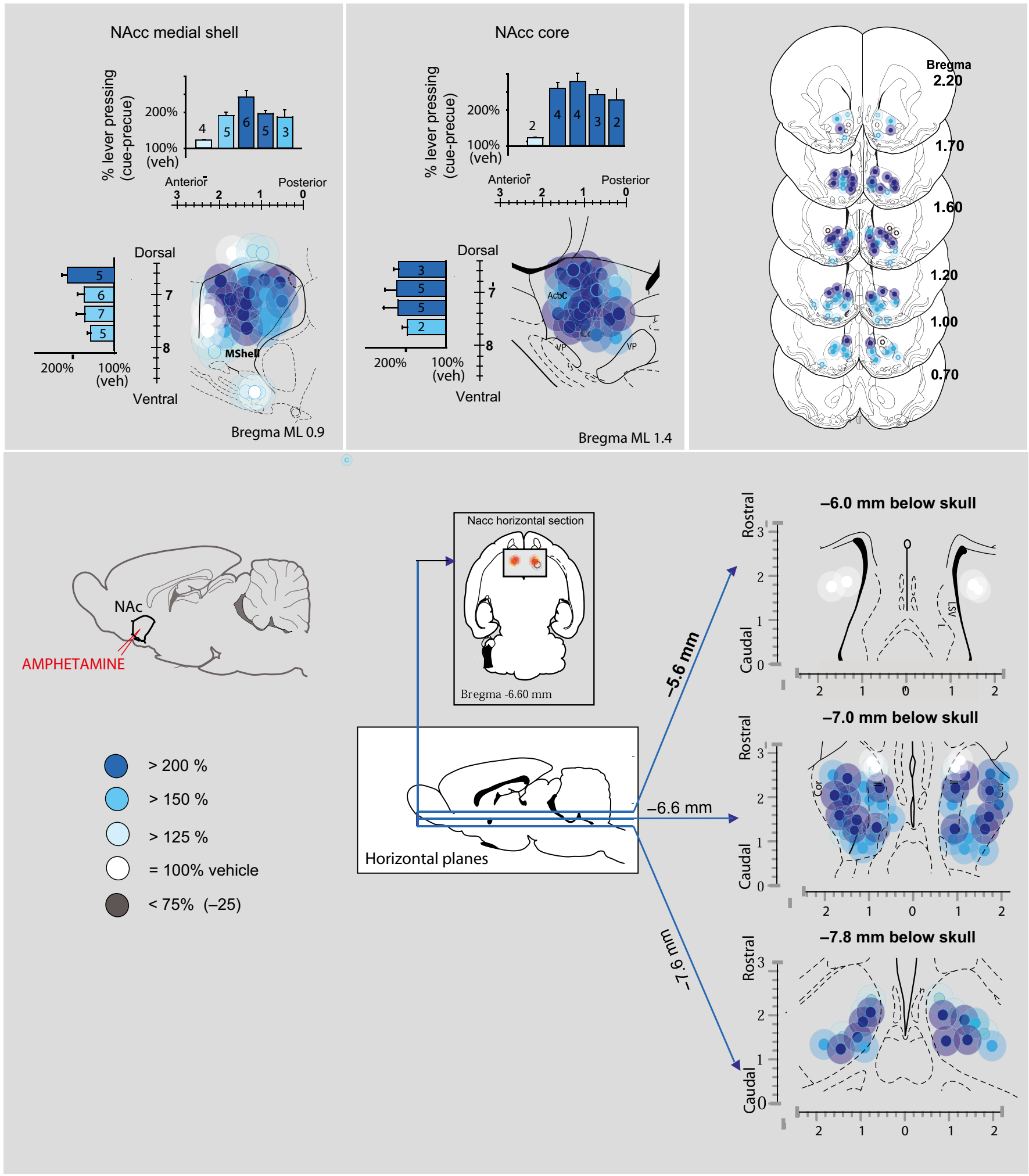

FIG. 3. Amphetamine maps of functional localization for cue-triggered 'wanting'. Fos plume maps show behavioral consequences of amphetamine microinjections specifically on CS+ triggered increases in lever pressing. The color intensity of the blue symbol depicts the behavioral intensity of PIT amplifications produced by amphetamine microinjection at that nucleus accumbens (NAc) site. Symbol size and color spread reflect the measured average diameter of microinjection Fos plumes produced by each drug. Maps are shown in sagittal planes (separate maps for shell and core) and coronal planes (top), and in three horizontal planes (to depict the maximum number of sites simultaneously in both the shell and core; bottom). The numerals in the bars depict the number of rats with sites at each stereotactic level. 

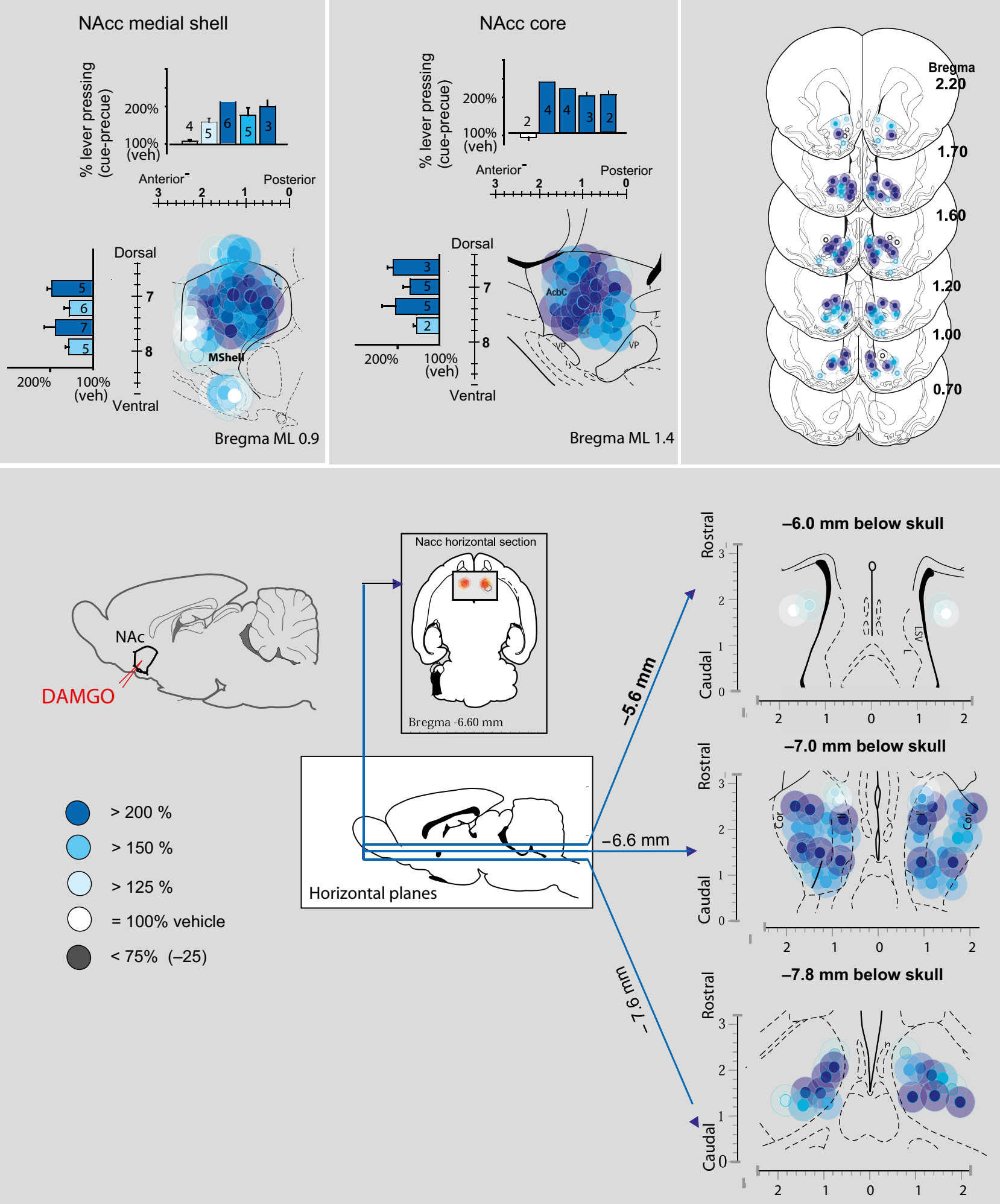

FIG. 4. DAMGO maps of functional localization for cue-triggered 'wanting'. Fos plume maps show behavioral consequences of DAMGO microinjections specifically on CS+ triggered increases in lever pressing. The color intensity of the blue symbol depicts the behavioral intensity of PIT amplifications produced by DAMGO microinjection at that nucleus accumbens (NAc) site. Symbol size and color spread reflect the measured average diameter of microinjection Fos plumes produced by each drug. Maps are shown in sagittal planes (separate maps for shell and core) and coronal planes (top), and in three horizontal planes (to depict the maximum number of sites simultaneously in both the shell and core; bottom). The numerals in the bars depict the number of rats with sites at each stereotactic level. 

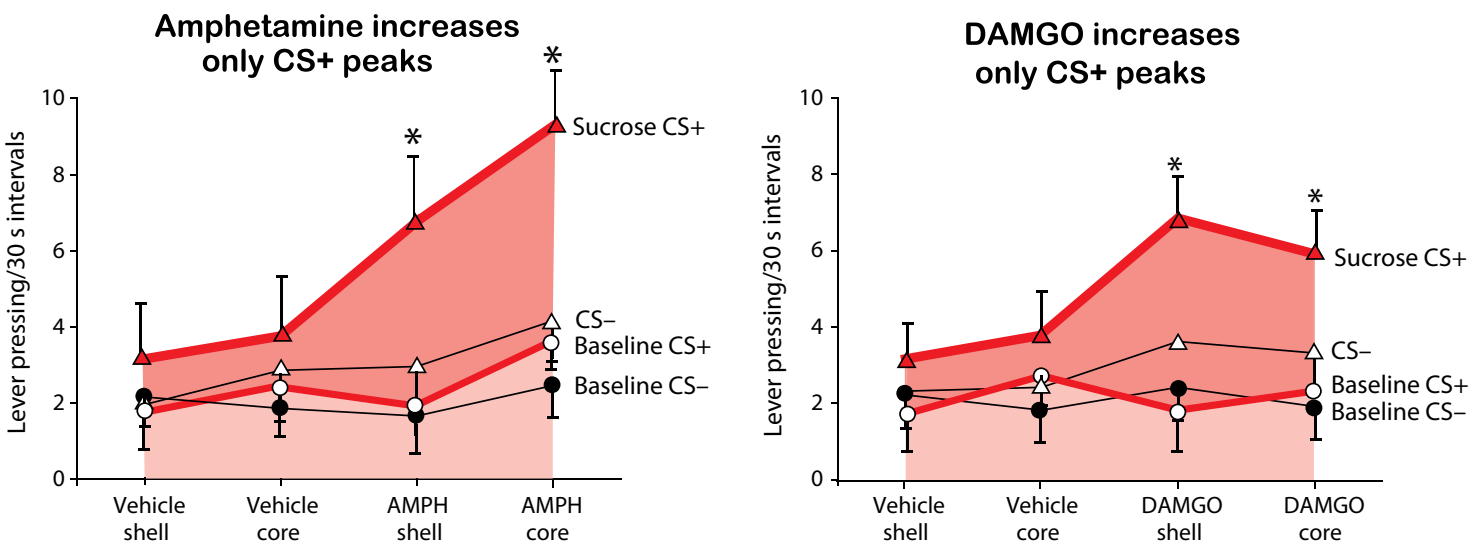

FIG. 5. Cue-by-cue pattern of lever pressing during the PIT test. The time course of pressing is shown during an entire 30-min extinction test session. The symbols show baseline lever pressing [pre-conditioned stimulus (CS) and post-CS 30-s bins], intense cue-triggered surges of lever pressing induced during the CS+ (sucrose cue; red) and in the immediately following 30-s bin (red), and the relative lack of effect of CS-. Amphetamine (top) or DAMGO (middle row) microinjections in the core (left) or medial shell (right) all dramatically amplified cue-triggered surges far above control vehicle levels (bottom).

\section{Amphetamine and DAMGO microinjections in the NAc shell and core increased PIT: cue-triggered elevations of lever pressing for sucrose in extinction}

Neurochemically in PIT effects, both amphetamine microinjections $(20 \mu \mathrm{g} / 0.2 \mu \mathrm{L})$ and DAMGO microinjections $(0.05 \mu \mathrm{g} / 0.2 \mu \mathrm{L})$ in the NAc comparably magnified the intensity of CS+-triggered pulses of increased pressing on the lever that had previously earned sucrose pellets, compared with vehicle levels. Neuroanatomically, sites throughout the NAc core and NAc medial shell were nearly all effective at generating drug-induced enhancements of PIT to levels between $200 \%$ and $250 \%(100 \%=$ normal vehicle PIT magnitude; amphetamine shell: $F_{1,86}=4.19, P<0.05$; core: $F_{1,62}=9.27, \quad P<0.05$; Fig. 3; DAMGO shell: $F_{1,80}=10.52$, $P<0.05$; core: $F_{1,64}=7.40, P<0.05$; Fig. 4 ), and the magnitude of enhancement did not differ across sites when compared statistically between the core and shell $(P=0.31$, n.s.). PIT enhancements were temporally expressed as phasic and temporary pulses of increased pressing on the active lever triggered by CS+ presentations, similar to normal PIT, but just with magnitudes approximately twice as high as normal. Each pulse of DAMGO/ amphetamine-induced amplification lasted approximately $1 \mathrm{~min}$, rising within seconds once the $\mathrm{CS}+$ sound began, remaining high during its 30-s auditory presentation, and persisting for about $30 \mathrm{~s}$ afterward while gradually decaying back to the baseline level (Fig. 5). That is, each drug produced comparable doubling of cuetriggered 'wanting' peaks at most NAc sites, but neither drug increased lever pressing at other baseline times during the session (when CS+ was absent), nor during presentations of the CSsound that predicted nothing (interaction of cue presence $\times$ amph: $F_{1,86}=17.83, P<0.001$; baseline: shell: $F_{1,86}=2.35, P=0.27$, n.s.; core: $F_{1,86}=1.86, P=0.18$, n.s.; Fig 3; $C S-$ presentations: shell DAMGO: $F_{1,86}=1.27, \quad P=0.65, \quad$ n.s.; core DAMGO: $F_{1,62}=1.53, P=0.85$, n.s.; Fig. 4). Finally, neither amphetamine nor DAMGO altered pressing on the control lever at any time [i.e. the lever that had never earned sucrose (though both levers were inactive in extinction during PIT tests)].

\section{Transfer score confirmation}

Another useful way of assessing PIT effects is to calculate transfer scores between lever pressing during the presentation of the Pavlov- ian cue $(\mathrm{CS}+)$ and during the baseline period. The transfer score is obtained by subtracting baseline pressing from $\mathrm{CS}+$ pressing on a within-subject basis, so that the remainder shows the enhancement during CS+ for each rat as a difference score. Transfer score results confirmed that amphetamine and DAMGO microinjections in the NAc shell and core produced similar amplifications in cue-triggered peaks of pressing (shell: $F_{1,40}=8.61, P<0.01$; core: $F_{1,32}=7.25$, $P<0.05$; Fig. 6). Shell microinjections of amphetamine increased cue-triggered peaks of lever pressing by $162 \%$ over vehicle levels, and core microinjections increased cue-triggered peaks by $179 \%$. Likewise, DAMGO microinjections in the NAc shell and core specifically amplified the incentive effect of the CS+ on pressing the sucrose-associated lever (shell: $F_{1,40}=8.61, \quad P<0.01$; core: $F_{1,32}=5.62, P<0.05$; Fig. 6).

\section{Anatomical detail on widespread enhancement network in medial shell and core}

Mapping localization of function within the NAc in behavioral causation maps confirmed that essentially all NAc sites successfully supported amphetamine amplifications in cue-triggered incentive salience (Fig. 3). That is, all individual microinjection sites between $2.5 \mathrm{~mm}$ and $0.7 \mathrm{~mm}$ anterior to bregma in the medial shell or core were equally effective at amplifying incentive salience. There was no evidence of localization for incentive salience enhancement in the form of discrete hotspots, anatomical rostrocaudal gradients or other localization patterns. The only exception to NAc site equipotentiality was the farthest most rostral edge of the medial shell and core where DAMGO failed to increase lever pressing peaks $(+2.0$ to $+2.5 \mathrm{~mm}$ from bregma; $F_{1,6}=1.12, P=0.25$, n.s.; Fig. 4 ). Differences in function have also been previously reported for the farthest rostral strip $(0.5 \mathrm{~mm}$ wide) compared with the rest of the medial shell (Peciña \& Berridge, 2000, 2005; Reynolds \& Berridge, 2002). We conclude that this far-rostral $0.5 \mathrm{~mm}$ edge of the NAc may be less effective at enhancing PIT, but that the remaining $85 \%$ of the NAc, both shell and core, are highly and equally able to magnify cue-triggered peaks of 'wanting' for reward in response to mu opioid or dopamine stimulation.

Of course, all these conclusions apply to the particular doses tested here, and differences might emerge between dopamine and opioid stimulation of PIT in future tests of full dose-response curves. However, at these doses, the similarity of the doubling 
enhancement pattern produced by amphetamine and DAMGO at most NAc sites in the shell or core is quite striking.

\section{Anatomical control sites}

At anatomical control sites in the olfactory tubercle, neostriatum (caudate/putatmen) or lateral shell, enhancements were largely absent. For example, in the olfactory tubercle, no enhancements in cue-triggered lever pressing were produced by microinjections of either amphetamine or DAMGO $\left(F_{1,4}=4.23, P=0.82\right.$, n.s.; DAMGO: $F_{1,4}=3.58, P=0.35$, n.s.). Similarly, amphetamine or DAMGO microinjections at most sites of the lateral shell also failed to increase cue-triggered pressing (amphetamine: $F_{1,8}=3.54$, $P=0.23$, n.s.; DAMGO: $F_{1,8}=2.56, P=0.42$, n.s.). Thus, we conclude that virtually all of the core and medial shell, but perhaps not the lateral shell or olfactory tubercle, may be able to mediate opioid or dopamine amplification of cue-triggered 'wanting' as assessed by PIT.

\section{Discussion}

Our results show that microinjections causing either dopamine (amphetamine) or mu opioid (DAMGO) stimulation of the NAc produce intense and equal amplifications of incentive salience in PIT, expressed as cue-triggered phasic peaks of increased efforts to obtain sucrose reward. Our results also show that most microinjection sites (each affecting about $0.5 \mathrm{~mm}$ volume of the NAc, as reflected by Fos plume radii) throughout the medial shell and core of the NAc can mediate these amplifications of cue-triggered incentive motivation, again to approximately equal degrees. Amphetamine
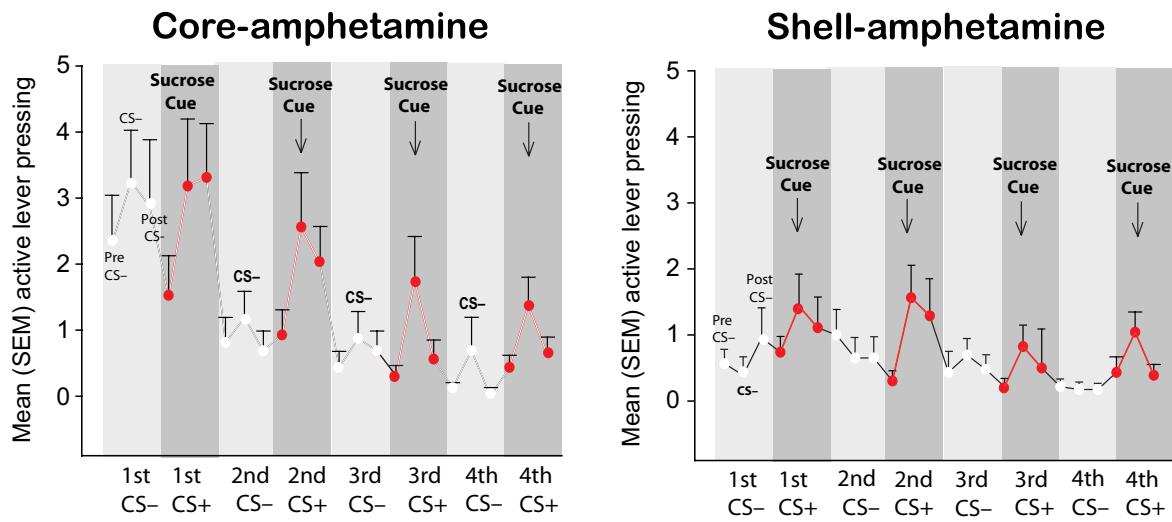

Core-DAMGO

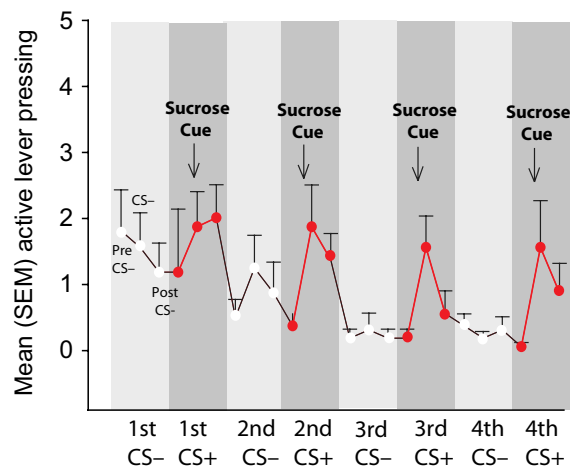

Core-vehicle

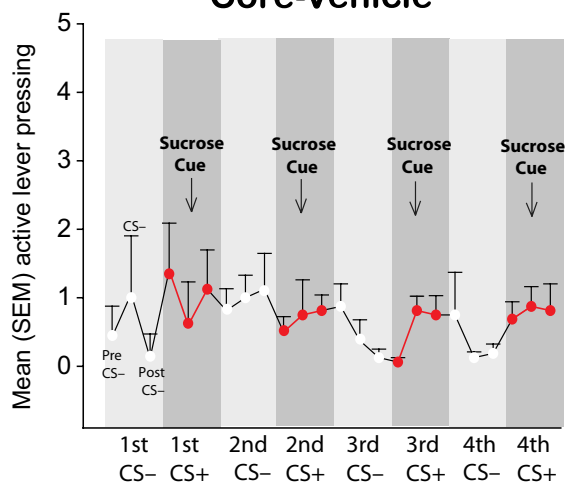

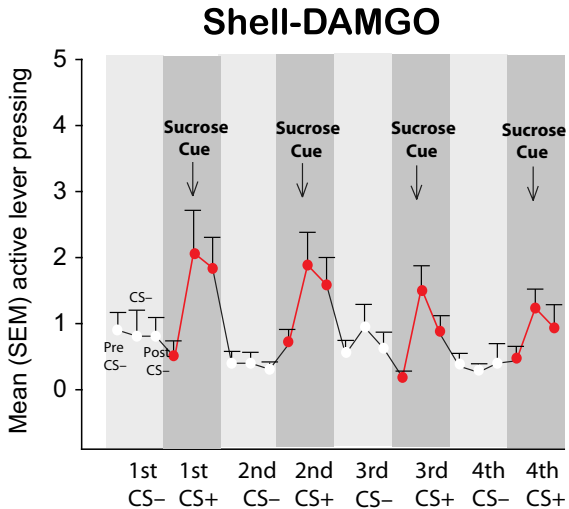

Shell-vehicle

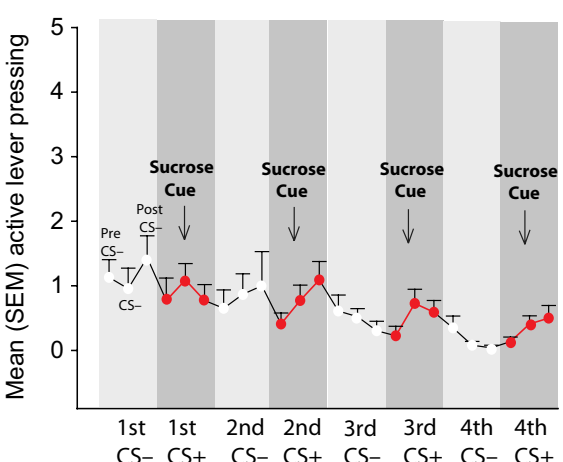

FIG. 6. Amplification of cue-triggered 'wanting'. Quantitative comparison of amphetamine and DAMGO for each microinjection and stimulus condition shows that only conditioned stimulus (CS) + moments were significantly amplified by shell or core microinjections. The relative magnitudes of CS + enhancements were roughly comparable for both drugs and sites. 
and DAMGO microinjections at nearly all sites within the entire core or within $90 \%$ of the medial shell directly and selectively magnified PIT peaks by $>150 \%$ (compared with vehicle control levels measured in the same individuals).

Amplification was always phasic, and restricted to the 1-min pulses of cue-triggered increase in motivation, evident behaviorally as temporary peaks of instrumental pressing whenever the 30-s CS+ cue was presented. This increase in reward-seeking behavior was always specifically directed to the lever that had previously earned sucrose pellets. Opioid and dopamine amplifications were dramatic whenever the CS+ was actually present and for about $30 \mathrm{~s}$ afterwards, but vanished during baseline and $\mathrm{CS}-$ periods when CS+ was absent. Once the CS+ ended, the amplified level of instrumental pressing quickly decayed back down to baseline levels no higher than vehicle levels (only to reappear again as drug-induced amplification on the next $\mathrm{CS}+$ re-encounter). That phasic nature of druginduced amplification demonstrates the synergistic interaction in incentive salience generation that exists between a Pavlovian CS+ and the neurobiological state of mesocorticolimbic systems at re-encounter (Zhang et al., 2009; Berridge, 2012).

\section{Equivalence of dopamine and opioid stimulation for cue-triggered 'wanting'}

The difference between dopamine and mu opioid effects in the NAc is not that opioids always cause increased 'liking' but not 'wanting', whereas dopamine causes 'wanting' but not 'liking'. As demonstrated here, opioid stimulation directly causes increased cue-triggered 'wanting' as well as dopamine stimulation, and at nearly all NAc sites. The pattern is not so simple as mutual exclusivity. The difference is that NAc opioid signals can additionally cause 'liking' as well as 'wanting' - but only if the opioid stimulation occurs in the $\mathrm{mm}^{3}$ hedonic hotspot located in the rostrocaudal quadrant of the medial shell (constituting about $10 \%$ of total NAc volume including core + shell; Peciña \& Berridge, 2005; Smith et al., 2011). 'Liking' enhancement does not occur at other subregions in the shell or at any known sites in the core (Peciña \& Berridge, 2005). The rostrodorsal quadrant of the medial shell has unique patterns of anatomical connectivity compared with other subregions of medial shell as well as the core, which might underlie its hedonic uniqueness (Peciña \& Berridge, 2005; Thompson \& Swanson, 2010; Zahm et al., 2012). Our results suggest that in nearly all the remainder of the NAc, mu opioid stimulation may have effects functionally identical to dopamine stimulation: both elevate 'wanting' (without needing to elevate 'liking'). By contrast, dopamine stimulation simply enhances 'wanting' without 'liking' at all NAc sites. Even in the NAc hotspot, dopamine behaves like mu opioid stimulation outside the hotspot.

Regarding anatomical localization, cue-triggered 'wanting' was doubled in magnitude here by opioid as well as by dopamine stimulation at nearly all sites within the core and the entire medial shell [with the opioid exception only of a narrow vertical $0.5-\mathrm{mm}$ strip located at the farthest-rostral edge of the NAc (shell and core), located at an A-P level that is actually anterior to the rostral border of the mid-rostral hotspot]. The far-rostral $15 \%$ strip of medial shell has produced unique behavioral results, different from those of the mid-rostral hotspot, in our previous microinjection studies too (Reynolds \& Berridge, 2002; Peciña \& Berridge, 2005; Faure et al., 2010), suggesting the far-rostral strip may also be somewhat unique in function compared with the rest of the NAc.

The ability of mu opioid stimulation to amplify incentive motivation largely throughout the NAc is consistent with reports that DAMGO microinjections at most sites in the core or medial shell (and even some sites in the neostriatum and central amygdala) stimulate increases in eating behavior and food intake, that core as well as shell sites support, as well as in Pavlovian approach and instrumental breakpoints in efforts to obtain food rewards (Mucha \& Iversen, 1986; Bakshi \& Kelley, 1993; Peciña \& Berridge, 2000; Ragnauth et al., 2000; Zhang \& Kelley, 2002; Zhang et al., 2003; Kim et al., 2004; Mahler \& Berridge, 2009; Taha et al., 2009; Katsuura et al., 2011; DiFeliceantonio \& Berridge, 2012). Similarly, dopamine ability to amplify PIT at most NAc sites is consistent with reports of dopamine involvement in both NAc core and shell, and in the neostriatum for similar behaviors (Volkow et al., 2004; Kelley et al., 2005; Peciña \& Berridge, 2005; Palmiter, 2007; Shiflett \& Balleine, 2010; Flagel et al., 2011; Saddoris et al., 2011).

However, we note that many previous lesion or blockade studies have reported differences between NAc core and medial shell manipulations regarding reward learning or Pavlovian-evoked motivation, including NAc lesion/blockade studies that produced PIT impairments (Voorn et al., 1989; Zahm \& Brog, 1992; Cardinal et al., 2002; Everitt \& Wolf, 2002; Di Chiara et al., 2004; Ikemoto \& Wise, 2004; Kelley, 2004; Shiflett \& Balleine, 2010; Ambroggi et al., 2011; Saddoris et al., 2011; Cacciapaglia et al., 2012; Chaudhri et al., 2012). With such previous reports of core vs. shell differences in mind, we emphasize that our 'widespread network' conclusion pertains only to neurochemical stimulation effects, and specifically to the amplification of incentive salience to higher-thannormal intensities (expressed here in PIT as elevated peak surges of cue-triggered 'wanting' for UCS). Regarding amplification of incentive salience, both the core and medial shell seem to contain substrates able to boost Pavlovian motivation. This conclusion may be compatible with evidence from previous lesion and pharmacological blockade studies showing that both the core and shell are required for at least some aspects of normal lower levels of PIT, even if conceivably in different aspects (Dickinson et al., 2000; Corbit et al., 2001; Hall et al., 2001; Lex \& Hauber, 2008; Shiflett \& Balleine, 2010; Saddoris et al., 2011). In short, we find widespread equipotentiality of NAc core and shell subregions for neurochemical enhancements of PIT, without evidence for hotspots or other localization of function.

\section{Modeling the synergy between CS+ and NAc state for incentive salience}

A mechanistic synergy in generating intense incentive salience is reflected in our observation of a need for simultaneous presence of physical CS+ and elevated NAc dopamine/opioid stimulation levels (which lasted at least for about $30 \mathrm{~min}$ after microinjection). In other words, neither by itself was sufficient for maximum peaks of 'wanting'. When the CS+ disappeared, the level of incentive salience declined within 1 min back to normal levels similar vehiclecontrol baselines, even when DAMGO or amphetamine stimulation remained in the brain. And while the CS+ always elicited phasic surges in 'wanting', the surges only attained maximal peak levels ( $>150 \%$ vehicle control peaks) when additional dopamine/opioid stimulation was simultaneously provided. Together the neurochemical-plus-CS + combinations were greater than the sum of their parts.

A computational model for describing such synergistic interaction in generating incentive salience was by suggested Zhang and colleagues as $\tilde{V}\left(S_{t}\right)=\tilde{r}\left(\kappa \bullet r_{t}\right)+\gamma V\left(S_{t+1}\right)$ (Zhang et al., 2009). That model captures well the synergy observed here. In the Zhang synergistic model $V\left(S_{t}\right)$ is the momentary intensity of incentive salience triggered at the time $t$ of encounter with the Pavlovian auditory CS+ or cue stimulus $(S)$. The Pavlovian association $\left(r_{t}\right)$ is based on 
previous pairings of CS+ with UCS (supplied in the Zhang model by a prediction error algorithm as a cached memory accumulation of previous UCS reward values), and the $\gamma$ factor is simply a temporal discounting parameter. Most important here is that the current neurobiological state of NAc circuitry, represented by the mesocorticolimbic gain factor $(\kappa)$ (i.e. kappa), reflects the effects of dopamine/ opioid receptor stimulation. The value of $\kappa$ essentially multiplies the previously learned $r_{t}$ value to generate incentive salience during the PIT test at the moment of CS+ re-encounter: $V\left(S_{t}\right)$. That motivation value was reflected in the phasic peak of cue-triggered 'wanting'.

To put our results in context, normal $\kappa$ was equal to 1 in our study as long as the NAc state during the test remained similar to states during previous training (i.e. in control test conditions after vehicle microinjection). By comparison, neurochemical stimulation of the NAc by amphetamine or DAMGO microinjections raised $\kappa$ during PIT tests to a quantity $>1$. That elevation in mesocorticolimbic reactivity raised the multiplicative product $\left(\kappa * r_{t}\right)$ of the interaction specifically at moments when $\mathrm{CS}+$ was physically present, elevating phasic peaks of PIT above normal levels.

Thus, incentive salience as $V\left(S_{t}\right)$ was specifically amplified selectively during $\mathrm{CS}+$ moments in the PIT tests after NAc drug microinjections. The pulses of amplified motivation came and went as phasic surges of cue triggered for UCS, coming and going with the physical CS+ stimulus, reflecting the synergistic interaction between the simultaneous presence of neurochemical stimulation and $\mathrm{CS}+$ presence.

\section{Applications and origins}

Reward cues often pose potent temptations, yet not always. The power of a reward CS+ to elicit motivated behavior varies from encounter to encounter. For example, an individual may successfully resist cue-triggered temptations on many occasions, yet still give in and fail to resist on another occasion. Fluctuation in cue motivation strength implies that the variation in temptation power lies in the dynamic reaction of brain mesocorticolimbic systems that interact with the cue, and not simply in the cue itself or in any stable learned association the CS+ has with its UCS. Our results indicate that dopamine or opioid stimulation of nearly any site in the medial shell and core can amplify this dynamic reaction, raising the intensity of incentive salience to generate a temporary cue-bound peak of maximal temptation.

The capacity for dynamic brain modulations of cue-triggered 'wanting' may have evolved originally as an adaption that let natural hunger or satiety states, which physiologically modulate mesocorticolimbic circuit reactivity, alter the level of cue-triggered incentive salience for relevant biological rewards, such as food, water, salt, mates, etc. (Kelley, 2004; Farooqi et al., 2007; Berthoud \& Morrison, 2008; Farooqi \& O'Rahilly, 2009; Kessler, 2009; Tindell et al., 2009; Zhang et al., 2009; Shin et al., 2010). However, once mesocorticolimbic circuits evolved to have capacities for synergistic interaction, such flexible capacity opens windows of vulnerability to addictive drugs and long-term changes involved in addiction to create compulsive levels of 'wanting' for particular incentives, such as drug-induced sensitization (Robinson \& Berridge, 1993, 2003, 2008; Volkow et al., 2004; Everitt \& Robbins, 2005; Berridge, 2012; Vanderschuren \& Pierce, 2010). This synergistic interaction allows higher peaks of temptation to be produced than would otherwise naturally occur.

We suggest that such motivation synergies as demonstrated here may help explain why the intensity of motivation elicited by a reward cue can fluctuate across time, and why addictive cues more potently trigger relapse on some encounters but not others. For example, an addict attempting to quit might successfully resist a drug cue many times again and even escape withdrawal - only to finally succumb to temptation and relapse on yet another catastrophic encounter. Our results suggest that potentiation of dopamine/opioid neurotransmission across a relatively wide region of the NAc might amplify incentive salience in this way (by any presence of drug on board or perhaps via endogenous emotional activation or stress states, etc.). In particular, the presence of any drug-on-board in a recovering addict who intended to take 'just one hit' could dangerously amplify cue-triggered 'wanting' to take more drugs. Synergistic generation of incentive salience by the NAc may thus be a potent mechanism to fuel the pathological pursuit of rewards at particular moments of most intense temptation.

\section{Acknowledgements}

This research was supported by grants from NIH (DA015188, and MH63649 to K.C. B.). We thank Michelle DiMondo for her assistance with Fos plume histology. The authors declare no conflict of interest.

\section{Abbreviations}

CR, conditioned response; CS, conditioned stimulus; DAMGO, d-Ala, nMePhe, Glyol-enkephalin; NAc, nucleus accumbens; PIT, Pavlovian-Instrumental Transfer; UCS, unconditioned stimulus; VI, variable.

\section{References}

Ambroggi, F., Ghazizadeh, A., Nicola, S.M. \& Fields, H.L. (2011) Roles of nucleus accumbens core and shell in incentive-cue responding and behavioral inhibition. J. Neurosci., 31, 6820-6830.

Bakshi, V.P. \& Kelley, A.E. (1993) Striatal regulation of morphine-induced hyperphagia: an anatomical mapping study. Psychopharmacology, 111, 207-214.

Baldo, B.A. \& Kelley, A.E. (2007) Discrete neurochemical coding of distinguishable motivational processes: insights from nucleus accumbens control of feeding. Psychopharmacology, 191, 439-459.

Barbano, M.F., Le Saux, M. \& Cador, M. (2009) Involvement of dopamine and opioids in the motivation to eat: influence of palatability, homeostatic state, and behavioral paradigms. Psychopharmacology, 203, 475-487.

Berridge, C.W., Stratford, T.L., Foote, S.L. \& Kelley, A.E. (1997) Distribution of dopamine beta-hydroxylase-like immunoreactive fibers within the shell subregion of the nucleus accumbens. Synapse, 27, 230-241.

Berridge, K.C. (2012) From prediction error to incentive salience: mesolimbic computation of reward motivation. Eur. J. Neurosci., 35, 1124-1143.

Berthoud, H.R. \& Morrison, C. (2008) The brain, appetite, and obesity. Annu. Rev. Psychol., 59, 55-92.

Bodnar, R.J. (2004) Endogenous opioids and feeding behavior: a 30-year historical perspective. Peptides, 25, 697-725.

Bray, S., Rangel, A., Shimojo, S., Balleine, B. \& O’Doherty, J.P. (2008) The neural mechanisms underlying the influence of pavlovian cues on human decision making. J. Neurosci., 28, 5861-5866.

Cacciapaglia, F., Saddoris, M.P., Wightman, R.M. \& Carelli, R.M. (2012) Differential dopamine release dynamics in the nucleus accumbens core and shell track distinct aspects of goal-directed behavior for sucrose. Neuropharmacology, 62, 2050-2056.

Cardinal, R.N., Parkinson, J.A., Hall, J. \& Everitt, B.J. (2002) Emotion and motivation: the role of the amygdala, ventral striatum, and prefrontal cortex. Neurosci. Biobehav. R., 26, 321-352.

Chaudhri, N., Sahuque, L.L., Schairer, W.W. \& Janak, P.H. (2012) Separable roles of the nucleus accumbens core and shell in context- and cue-induced alcohol-seeking. Neuropsychopharmacol., 35, 783-791.

Corbit, L.H. \& Balleine, B.W. (2005) Double Dissociation of Basolateral and Central Amygdala Lesions on the General and Outcome-Specific Forms of Pavlovian-Instrumental Transfer. J. Neurosci., 25, 962-970.

Corbit, L.H., Muir, J.L. \& Balleine, B.W. (2001) The role of the nucleus accumbens in instrumental conditioning: Evidence of a functional dissociation between accumbens core and shell. J. Neurosci., 21, 3251-3260. 
Corbit, L.H., Janak, P.H. \& Balleine, B.W. (2007) General and outcomespecific forms of Pavlovian-instrumental transfer: the effect of shifts in motivational state and inactivation of the ventral tegmental area. Eur. J. Neurosci., 26, 3141-3149.

Crombag, H.S., Galarce, E.M. \& Holland, P.C. (2008) Pavlovian influences on goal-directed behavior in mice: the role of cue-reinforcer relations. Learn. Memory, 15, 299-303.

Di Chiara, G., Bassareo, V., Fenu, S., De Luca, M.A., Spina, L., Cadoni, C., Acquas, E., Carboni, E., Valentini, V. \& Lecca, D. (2004) Dopamine and drug addiction: the nucleus accumbens shell connection. Neuropharmacology, 47, 227-241.

Dickinson, A., Smith, J. \& Mirenowicz, J. (2000) Dissociation of Pavlovian and instrumental incentive learning under dopamine antagonists. Behav. Neurosci., 114, 468-483.

DiFeliceantonio, A.G. \& Berridge, K.C. (2012) Which cue to 'want'? Opioid stimulation of central amygdala makes goal-trackers show stronger goaltracking, just as sign-trackers show stronger sign-tracking. Behav. Brain Res., 230, 399-408.

Estes, W.K. (1943) Discriminative conditioning. I. A discriminative property of conditioned anticipation. J. Exp. Psychol., 32, 150-155.

Everitt, B.J. \& Robbins, T.W. (2005) Neural systems of reinforcement for drug addiction: from actions to habits to compulsion. Nat. Neurosci., 8 , 1481-1489.

Everitt, B.J. \& Wolf, M.E. (2002) Psychomotor stimulant addiction: A neural systems perspective. J. Neurosci., 22, 3312-3320.

Farooqi, I.S. \& O'Rahilly, S. (2009) Leptin: a pivotal regulator of human energy homeostasis. Am. J. Clin. Nutr., 89, 980S-984S.

Farooqi, I.S., Bullmore, E., Keogh, J., Gillard, J., O'Rahilly, S. \& Fletcher, P.C. (2007) Leptin regulates striatal regions and human eating behavior. Science, 317, 1355

Faure, A., Richard, J.M. \& Berridge, K.C. (2010) Desire and dread from the nucleus accumbens: cortical glutamate and subcortical GABA differentially generate motivation and hedonic impact in the rat. PLOS ONE, 5, e11223.

Flagel, S.B., Clark, J.J., Robinson, T.E., Mayo, L., Czuj, A., Willuhn, I., Akers, C.A., Clinton, S.M., Phillips, P.E. \& Akil, H. (2011) A selective role for dopamine in stimulus-reward learning. Nature, 469, 53-57.

Hall, J., Parkinson, J.A., Connor, T.M., Dickinson, A. \& Everitt, B.J. (2001) Involvement of the central nucleus of the amygdala and nucleus accumbens core in mediating Pavlovian influences on instrumental behaviour. Eur. J. Neurosci., 13, 1984-1992.

Holland, P.C. (2004) Relations between Pavlovian-instrumental transfer and reinforcer devaluation. J. Exp. Psychol. Anim. B., 30, 104-117.

Holland, P.C. \& Gallagher, M. (2003) Double dissociation of the effects of lesions of basolateral and central amygdala on conditioned stimulus-potentiated feeding and Pavlovian-instrumental transfer. Eur. J. Neurosci., 17, $1680-1694$

Ikemoto, S. (2007) Dopamine reward circuitry: two projection systems from the ventral midbrain to the nucleus accumbens-olfactory tubercle complex. Brain Res. Rev., 56, 27-78.

Ikemoto, S. \& Wise, R.A. (2004) Mapping of chemical trigger zones for reward. Neuropharmacology, 47(Suppl 1), 190-201.

Kelley, A.E. (2004) Ventral striatal control of appetitive motivation: role in ingestive behavior and reward-related learning. Neurosci. Biobehav. R., 27, 765-776.

Kelley, A.E., Baldo, B.A., Pratt, W.E. \& Will, M.J. (2005) Corticostriatalhypothalamic circuitry and food motivation: Integration of energy, action and reward. Physiol. Behav., 86, 773-795.

Kessler, D.A. (2009) The End of Overeating: Taking Control of the Insatiable American Appetite. Rodale Press (Macmillan), New York.

Kim, E.M., Quinn, J.G., Levine, A.S. \& O'Hare, E. (2004) A bi-directional mu-opioid-opioid connection between the nucleus of the accumbens shell and the central nucleus of the amygdala in the rat. Brain Res., 1029, 135139.

Laurent, V., Leung, B., Maidment, N. \& Balleine, B.W. (2012) $\mu$ - and $\delta$ Opioid-Related Processes in the Accumbens Core and Shell Differentially Mediate the Influence of Reward-Guided and Stimulus-Guided Decisions on Choice. J. Neurosci., 32, 1875-1883.

Levine, A.S. \& Billington, C.J. (2004) Opioids as agents of reward-related feeding: a consideration of the evidence. Physiol. Behav., 82, 57-61.

Lex, A. \& Hauber, W. (2008) Dopamine D1 and D2 receptors in the nucleus accumbens core and shell mediate Pavlovian-instrumental transfer. Learn. Memory, 15, 483-491.

Mahler, S.V. \& Berridge, K.C. (2009) Which cue to "want?" Central amygdala opioid activation enhances and focuses incentive salience on a prepotent reward cue. J. Neurosci., 29, 6500-6513.
Mucha, R.F. \& Iversen, S.D. (1986) Increased food intake after opioid microinjections into nucleus accumbens and ventral tegmental area of rat Brain Res., 397, 214-224.

O'Connor, E.C., Stephens, D.N. \& Crombag, H.S. (2010) Modeling appetitive Pavlovian-instrumental interactions in mice. Curr. Protoc. Neurosci. $\mathbf{8}, 8.25$.

Ostlund, S.B. \& Maidment, N.T. (2012) Dopamine Receptor Blockade Attenuates the General Incentive Motivational Effects of Noncontingently Delivered Rewards and Reward-Paired Cues Without Affecting Their Ability to Bias Action Selection. Neuropsychopharmacol., 37, 508-519.

Palmiter, R.D. (2007) Is dopamine a physiologically relevant mediator of feeding behavior? Trends Neurosci., 30, 375-381.

Paxinos, G. \& Watson, C. (1996) The rat brain in stereotaxic coordinates. Academic Press, New York.

Peciña, S. \& Berridge, K.C. (2000) Opioid eating site in accumbens shell mediates food intake and hedonic 'liking': map based on microinjection Fos plumes. Brain Res., 863, 71-86.

Peciña, S. \& Berridge, K.C. (2005) Hedonic hot spot in nucleus accumbens shell: Where do mu-opioids cause increased hedonic impact of sweetness? J. Neurosci., 25, 11777-11786.

Peciña, S., Schulkin, J. \& Berridge, K.C. (2006) Nucleus accumbens corticotropin-releasing factor increases cue-triggered motivation for sucrose reward: paradoxical positive incentive effects in stress? BMC Biol., 4, 8 .

Pielock, S.M., Lex, B. \& Hauber, W. (2011) The role of dopamine in the dorsomedial striatum in general and outcome-selective Pavlovian-instrumental transfer. Eur. J. Neurosci., 33, 717-725.

Ragnauth, A., Moroz, M. \& Bodnar, R.J. (2000) Multiple opioid receptors mediate feeding elicited by mu and delta opioid receptor subtype agonists in the nucleus accumbens shell in rats. Brain Res., 876, 76-87.

Reynolds, S.M. \& Berridge, K.C. (2002) Positive and negative motivation in nucleus accumbens shell: Bivalent rostrocaudal gradients for GABA-elicited eating, taste "liking"/"disliking" reactions, place preference/avoidance, and fear. J. Neurosci., 22, 7308-7320.

Reynolds, S.M. \& Berridge, K.C. (2008) Emotional environments retune the valence of appetitive versus fearful functions in nucleus accumbens. Nat. Neurosci., 11, 423-425.

Richard, J.M. \& Berridge, K.C. (2011) Metabotropic glutamate receptor blockade in nucleus accumbens shell shifts affective valence towards fear and disgust. Eur. J. Neurosci., 33, 736-747.

Robinson, T.E. \& Berridge, K.C. (1993) The neural basis of drug craving: an incentive-sensitization theory of addiction. Brain Res. Rev., 18, 247291.

Robinson, T.E. \& Berridge, K.C. (2003) Addiction. Annu. Rev. Psychol., 54, $25-53$.

Robinson, T.E. \& Berridge, K.C. (2008) Review. The incentive sensitization theory of addiction: some current issues. Philos. T. Roy. Soc. B., 363 3137-3146.

Saddoris, M.P., Stamatakis, A. \& Carelli, R.M. (2011) Neural correlates of Pavlovian-to-instrumental transfer in the nucleus accumbens shell are selectively potentiated following cocaine self-administration. Eur. J. Neurosci., 33, 2274-2287.

Saunders, B.T. \& Robinson, T.E. (2012) The role of dopamine in the accumbens core in the expression of Pavlovian-conditioned responses. Eur. J. Neurosci., 36, 2521-32.

Shiflett, M.W. \& Balleine, B.W. (2010) At the limbic-motor interface: disconnection of basolateral amygdala from nucleus accumbens core and shell reveals dissociable components of incentive motivation. Eur. J. Neurosci., 32, 1735-1743.

Shin, A.C., Pistell, P.J., Phifer, C.B. \& Berthoud, H.R. (2010) Reversible suppression of food reward behavior by chronic mu-opioid receptor antagonism in the nucleus accumbens. Neuroscience, 170, 580-588.

Smith, K.S. \& Berridge, K.C. (2005) The ventral pallidum and hedonic reward: neurochemical maps of sucrose "liking" and food intake. J. Neurosci., 25, 8637-8649.

Smith, K.S., Berridge, K.C. \& Aldridge, J.W. (2011) Disentangling pleasure from incentive salience and learning signals in brain reward circuitry. Proc. Natl. Acad. Sci. USA, 108, 255-264.

Taha, S.A., Katsuura, Y., Noorvash, D., Seroussi, A. \& Fields, H.L. (2009) Convergent, not serial, striatal and pallidal circuits regulate opioid-induced food intake. Neuroscience, 161, 718-733.

Talmi, D., Seymour, B., Dayan, P. \& Dolan, R. (2008) Human pavlovianinstrumental transfer. J. Neurosci., 28, 360-368.

Thompson, R.H. \& Swanson, L.W. (2010) Hypothesis-driven structural connectivity analysis supports network over hierarchical model of brain architecture. Proc. Natl. Acad. Sci. USA, 107, 15235-15239. 
Tindell, A.J., Smith, K.S., Berridge, K.C. \& Aldridge, J.W. (2009) Dynamic computation of incentive salience: "wanting" what was never "liked". J. Neurosci., 29, 12220-12228.

Van Ree, J.M., Niesink, R.J., Van Wolfswinkel, L., Ramsey, N.F., Kornet, M.M. Van Furth, W.R., Vanderschuren, L.J., Gerrits, M.A. \& Van den Berg, C.L. (2000) Endogenous opioids and reward. Eur. J. Pharmacol., 405, 89-101.

Vanderschuren, L.J. \& Pierce, R.C. (2010) Sensitization processes in drug addiction. Curr. Top. Behav. Neurosci., 3, 179-195.

Volkow, N.D., Fowler, J.S. \& Wang, G.J. (2004) The addicted human brain viewed in the light of imaging studies: brain circuits and treatment strategies. Neuropharmacology, 47, 3-13.

Voorn, P., Gerfen, C.R. \& Groenewegen, H.J. (1989) Compartmental organization of the ventral striatum of the rat: immunohistochemical distribution of enkephalin, substance $\mathrm{P}$, dopamine, and calcium-binding protein J. Comp. Neurol., 289, 189-201.

Walker, K.C. (1942) The effect of a discriminative stimulus transferred to a previously unassociated response. J. Exp. Psychol., 31, 312-321.

Wassum, K.M., Ostlund, S.B., Balleine, B.W. \& Maidment, N.T. (2011) Differential dependence of Pavlovian incentive motivation and instrumental incentive learning processes on dopamine signaling. Learn. Memory, 18, 475-483.

Wyvell, C.L. \& Berridge, K.C. (2000) Intra-accumbens amphetamine increases the conditioned incentive salience of sucrose reward: enhance- ment of reward "wanting" without enhanced "liking" or response reinforcement. J. Neurosci., 20, 8122-8130.

Wyvell, C.L. \& Berridge, K.C. (2001) Incentive-sensitization by previous amphetamine exposure: Increased cue-triggered 'wanting' for sucrose reward. J. Neurosci., 21, 7831-7840.

Zahm, D.S. \& Brog, J.S. (1992) On the significance of subterritories in the "accumbens" part of the rat ventral striatum. Neuroscience, 50, 751 $-767$.

Zahm, D.S., Parsley, K.P., Schwartz, Z.M. \& Cheng, A.Y. (2012) On lateral septum-like characteristics of outputs from the accumbal hedonic 'hotspot' of Pecina and Berridge with commentary on the transitional nature of basal forebrain 'boundaries'. J. Comp. Neurol., 521, 50-68.

Zhang, M. \& Kelley, A.E. (2002) Intake of saccharin, salt, and ethanol solutions is increased by infusion of a mu opioid agonist into the nucleus accumbens. Psychopharmacology, 159, 415-423.

Zhang, M., Balmadrid, C. \& Kelley, A.E. (2003) Nucleus accumbens opioid, GABaergic, and dopaminergic modulation of palatable food motivation: contrasting effects revealed by a progressive ratio study in the rat. Behav. Neurosci., 117, 202-211.

Zhang, J., Berridge, K.C., Tindell, A.J., Smith, K.S. \& Aldridge, J.W. (2009) A neural computational model of incentive salience. PLoS Comput. Biol., 5, e1000437. 